\title{
Newtons Gravitational Field Equations for a Static Homogeneous Spherical Distribution of Mass in Rotational Spherical Polar Coordinates
}

\author{
Obagboye, L. $\mathrm{F}^{1}$;Howusu,S.X.K ${ }^{2}$ \\ ${ }^{1}$ Theoretical Physics Programme, National Mathematical Centre Abuja P.M.B 118 Garki P.O. \\ ${ }^{2}$ Physics Department, Kogi State University Anyigba Kogi State, Nigeria.
}

Abstract: In this paper we formulate and solve Newton's gravitational Field equations for a static homogeneous spherical distribution of mass in rotational spherical polar coordinates to pave the way for applications such as planetary theory in rotational spherical polar coordinates.

Key Words: Newton's Gravitational Field Equations, Static Homogeneous Spherical distribution of mass, Rotational Spherical Polar Coordinates

Theory

It is well established that the Newton's gravitational Field equations for the gravitational scalar potential $f$ due to a distribution of mass density $\rho$ is given by

$\nabla^{2}\left(x^{\mu}\right)=4 \pi G \rho_{0}\left(x^{\mu}\right)^{[1]}$

where $\nabla^{2}$ is the Euclidean Laplacian given by ${ }^{[2]}$

$$
\begin{gathered}
\nabla^{2}=\frac{1}{h_{1} h_{2} h_{3}}\left\{\frac{\partial}{\partial u}\left[\frac{h_{2} h_{3}}{h_{1}} \frac{\partial}{\partial u}\right]+\frac{\partial}{\partial v}\left[\frac{h_{1} h_{3}}{h_{2}} \frac{\partial}{\partial v}\right]+\frac{\partial}{\partial w}\left[\frac{h_{1} h_{2}}{h_{3}} \frac{\partial}{\partial w}\right]\right\} \\
h_{1} h_{2} h_{3} \text { are scale factors }
\end{gathered}
$$

It follows immediately that

$$
\begin{aligned}
& \nabla^{2}=\frac{1}{\left[\frac{\mathrm{u}^{2}}{1-\mathrm{v}^{2}}\right]^{\frac{1}{2}}\left[\frac{\mathrm{u}\left(1-\mathrm{v}^{2}\right)}{1-\mathrm{w}^{2}}\right]^{\frac{1}{2}}} \\
& \left\{\left[\frac{\partial}{\partial u}\left[\frac{u^{2}}{1-v^{2}}\right]^{\frac{1}{2}}\left[\frac{u\left(1-v^{2}\right.}{1-w^{2}}\right]^{\frac{1}{2}} \frac{\partial}{\partial u}\right]+\frac{\partial}{\partial v}\left[\left[\frac{u\left(1-v^{2}\right.}{1-w^{2}}\right]^{\frac{1}{2}} \frac{\partial}{\partial v}\right]+\frac{\partial}{\partial w}\left[\frac{u^{2}}{\left(1-v^{2}\right)^{\frac{1}{2}}}\left[\frac{1-w^{2}}{u\left(1-v^{2}\right.}\right]^{\frac{1}{2}} \frac{\partial}{\partial w}\right]\right\}
\end{aligned}
$$

This reduces to

$$
\nabla^{2}=\frac{1}{u^{2}} \frac{\partial}{\partial u}\left(u^{2}\right)+\frac{1}{u} \frac{\partial}{\partial v}\left(\left(1-v^{2}\right) \frac{\partial}{\partial v}\right)+\frac{1}{u\left(1-v^{2}\right)} \frac{\partial}{\partial w}\left[\left(1-w^{2}\right) \frac{\partial}{\partial w}\right]
$$

\section{Research Elaborations}

For a Static Homogeneous Spherical distribution of mass in Rotational spherical polar coordinates we consider two conditions following from equation (1)

$$
\begin{aligned}
& \frac{1}{u^{2}} \frac{d}{d u}\left(u^{2} \frac{d}{d u}\right) f(u)=4 \pi G \rho_{0} ; u<R \\
& \frac{1}{u^{2}} \frac{d}{d u}\left(u^{2} \frac{d}{d u}\right) f(u)=0 ; u>R \\
& \text { From equation }(6) \\
& \frac{d}{d u}\left(u^{2} \frac{d}{d u} f\right)=0 \\
& u^{2} \frac{d}{d u} f=A \\
& \frac{d f}{d u}=\frac{A}{u^{2}} \\
& \Rightarrow f^{+}=-\frac{A}{U}+B
\end{aligned}
$$$$
\frac{1}{u^{2}} \frac{d}{d u}\left(u^{2} \frac{d}{d u}\right) f(u)=0 ; u>R
$$

From equation (5)

$$
\begin{aligned}
& \frac{1}{u^{2}} \frac{d}{d u}\left(u^{2} \frac{d}{d u}\right) f(u)=4 \pi G \rho_{0} \\
& \frac{d}{d u}\left(u^{2} \frac{d}{d u}\right) f(u)=\rho_{0} G \rho_{0} u^{2} \\
& \mathrm{u}^{2} \frac{\mathrm{d}}{\mathrm{du}} \mathrm{f}(\mathrm{u})=\frac{4}{3} \pi G \rho_{0} \mathrm{u}^{3}+\mathrm{A}
\end{aligned}
$$


$f(u)=\frac{4}{3} \pi G \rho_{0} \int u d u$

$f_{p}^{-}(u)=\frac{2}{3} \pi G \rho_{0} u^{2}+\mathrm{B}$

Solving for Complimentary $f^{-}{ }_{c}$

$\nabla^{2} f^{-}{ }_{c}=o$

$\frac{d}{d u}\left(u^{2} \frac{d}{d u} f^{-}{ }_{c}\right)=0$

Setting, $f_{c}^{-}=D U^{m}$

It follows that $m=-1$ or 0

$\Rightarrow f^{-}{ }_{c}=D$

$f^{-}=f^{-}{ }_{p}+f^{-}{ }_{c}$

$$
=D+\frac{2}{3} \pi G \rho_{0} u^{2}
$$

Knowing that $f^{+}=\frac{A}{U} ; U>R$ and also that $\rho_{0}=\frac{3 M_{o}}{4 \pi R^{3}}$

Therefore

$f^{-}=D+\frac{G M_{o} u^{2}}{2 R^{3}} ; U<R$

$f^{+}=\frac{A}{U} ; U>R$

Now applying the conditions $f^{-}=f^{+} ; U=R$ and $\frac{\partial f^{-}}{\partial u}=\frac{\partial f^{+}}{\partial u} ; U=R$ we have

$\frac{\partial}{\partial u}=\left[D+\frac{G M_{o} U^{2}}{2 R^{3}}\right]=\frac{\partial}{\partial u}\left[\frac{A}{U}+B\right]$

$\Rightarrow A=-G M_{O}$

From $f^{-}=f^{+}$we get

$D+\frac{G M_{o} U^{2}}{2 R^{3}}=\frac{A}{U}$

$\therefore D=\frac{G M_{O}}{2 R}$

The explicit Newton's gravitational Scalar potential field for a static homogeneous spherical distribution of mass is hence given by:

$f^{+}=\frac{G M_{0}}{U}$

$f^{-}=\frac{G M_{0}}{2 R}\left[1+\frac{U^{2}}{R^{2}}\right]$

\section{Conclusion}

The Scalar potential field so derived can now be applied to derive the Riemannian gravitational intensity for the interior and exterior fields and hence decompose the Riemann's geodesic equation ${ }^{[3]}$ into the Riemann's acceleration part and a pure gravitational intensity or acceleration due to gravity . The scalar potential field plays a fundamental role in establishing a proof for the vanishing of the Riemannian curvature scalar in the Newton's gravitational field exterior to a static homogeneous spherical distribution of mass which has far reaching consequences in the theories of gravitation.

\section{References}

[1] D.D. Bakwa and S.X.K. Howusu " Galaxies as a source of Gravitational Radiation", African Journal of Natural sciences, 6 pp 15 18

[2] M.R. Spiegel, Theory and Problems of Vector Analysis and Introduction to Tensor Analysis (Mc Graw - Hill, New York, 1974 ) 55

[3] S.X.K Howusu. 2013; Riemannian Revolution in Physics and Mathematics Jos University press Ltd. Jos pp103-115. 